Voix et Images

volxetimages

\title{
Des actes de langage à la poétique
}

\section{Jean Fisette}

Volume 11, numéro 3 (33), printemps 1986

Yolande Villemaire

URI : https://id.erudit.org/iderudit/200589ar

DOI : https://doi.org/10.7202/200589ar

Aller au sommaire du numéro

Éditeur(s)

Université du Québec à Montréal

ISSN

0318-9201 (imprimé)

1705-933X (numérique)

Découvrir la revue

Citer cet article

Fisette, J. (1986). Des actes de langage à la poétique. Voix et Images, 11(3),

547-550. https://doi.org/10.7202/200589ar d'utilisation que vous pouvez consulter en ligne.

https://apropos.erudit.org/fr/usagers/politique-dutilisation/ 


\title{
Des actes de langage à la poétique
}

\author{
par Jean Fisette, Université du Québec à Montréal
}

Le questionnement sur les formes littéraires, sur les actes d'énonciation, sur les signes et les théories qui les soutiennent constituent depuis plusieurs années des terrains d'élection dans le monde universitaire des Lettres. Après quelques années d'attente, voici que, coup sur coup, l'on reçoit deux ouvrages fort sérieux qui viennent, chacun à sa façon, attester de la vitalité du travail qui se poursuit dans l'ombre.

Dans les Signes s'envolent ${ }^{1}$, Louis Francoeur propose une construction théorique fondée sur la théorie des actes du langage et qui vise à donner rien de moins qu'une interprétation des conditions d'élaboration d'une culture, d'où le sous-titre: "Pour une sémiotique des actes de langage culturels".

Considérer les objets de la littérature sous le point de vue de la pragmatique, c'est nécessairement ramener l'œuvre à un statut de médium, de moyen de communication entre un destinateur et un destinataire; et c'est aussi pos- 
tuler chez l'auteur une intention d'agir directement sur son lecteur, devenu interlocuteur. À la limite, les.œuvres sont considérées, dans leur diversité, comme des variantes dans les moyens techniques mis en œuvre pour l'accomplissement de ces réseaux d'influence.

L'ensemble de ces réseaux d'influence, dans leurs interactions, leurs contradictions mêmes, voilà le substrat systémique qui fonde une culture.

Les études littéraires, qui depuis 25 ans n'ont pas hésité à aller fort loin dans la théorisation de leur objet, ont presque toujours évité cette perspective de la communication; voire plus, elles en ont souvent rejeté la validité affirmant que l'œuvre, une fois constituée, se détache de son auteur et entame une vie autonome produisant, à la rencontre des diverses lectures, des effets de sens imprévisibles.

En ce sens, L. Francoeur opère un revirement important, déplaçant la problématique littéraire, du lieu de la signification à celui de la communication. Ce déplacement me paraît si fondamental que je ne puis que regretter l'absence d'une introduction ou même d'un chapitre qui soit consacré à cette question théorique.

Les Signes s'envolent se construit au long de ses cinq chapitres sur deux principes de distribution des matières: d'abord un découpage suivant les genres littéraires: la nouvelle littéraire, le récit du monologue intérieur, le texte institutionnel du $\mathrm{XIX}^{\mathrm{e}}$ siècle, le théâtre et le récit de littérature orale. D'autre part, ces analyses dans leur succession produisent un effet cumulatif dans l'élaboration de la théorie des actes de langage appliquée au texte littéraire.

Ainsi dans le premier chapitre, sont énumérées les conditions nécessaires et suffisantes à l'accomplissement de l'acte illocutionnaire de narration (p. 24). Lorsque ces conditions (qu'il n'y a pas lieu de reprendre ici) sont respectées, le récepteur du récit peut être considéré comme un lecteur idéal, la communication a été pleine et entière, l'intention de l'auteur a atteint le lecteur. Et corrélativement, les manques à ces conditions, qui en fait sont des carences dans le codage du texte, peuvent expliquer qu'un texte n'atteigne pas son public lecteur.

Le second chapitre constitue, à mon avis, l'étude la mieux conduite. Après avoir rappelé la definition de l'information, non comme contenu, mais comme mesure d'une quantité d'originalité et d'imprévisibilité (p. 41), il est proposé de faire la distinction entre l'information dénotative, régie par des règles universelles ( $p .42$ ), soit les codes dominants dans l'ordre de la communication, alors que l' «information connotative" renvoie aux investissements les plus personnalisés de l'auteur. Dès lors, le récit à monologue intérieur est situé à mi-chemin entre la poésie lyrique (nette prédominance de l'information connotative) et le récit classique (nette prédominance de l'information dénotative). Ou, plus justement, les traits appartenant à la logique de l'information connotative, en obscurcissant la vision, permettent de rendre la sensation de la vie (p. 63). 
Au troisième chapitre, les textes analysés, tous puisés au répertoire du dernier siècle, se caractérisent par leurs marques très visibles d'appartenance à des débats idéologiques qui se tenaient autrefois et qui n'ont plus cours. Se pose alors, inévitablement, la question de la valeur esthétique. Francoeur oppose à ces interrogations la nécessité de resituer ces œuvres dans les séries culturelles auxquelles elles ont donné naissance de sorte que ces textes quelque peu vieillis sont ainsi réintégrés dans un questionnement contemporain.

Le chapitre suivant pose le théâtre comme la représentation d'une communication. Tout le débat soulevé tourne alors autour de la question de l'acte de représentation: ainsi le rapport d'identification que le spectateur établit avec un personnage prend' les allures d'un réseau d'échange qui avait été programmé par l'auteur. Mais pour que l'acte illocutoire se réalise, il faut que l'acte de langage (...) transforme l'interaction de la dyade scène-salle de complémentaire qu'elle était auparavant la scène occupant par convention dans la série culturelle la position haute et la salle la position basse) en interaction symétrique (p. 124). Dans ce cadre théorique, il est tout à fait logique que l'analyse s'intéresse à des pièces comme Médium saignant et les Fées ont soif.

Le dernier chapitre procède à une analyse comparée de deux contes merveilleux tirés de la littérature orale: études extrêmement minutieuses suivant un modèle inspiré des études de structuration narrative. Dans ce genre, comme dans les autres, l'acte de raconter devient un acte illocutoire de production d'effet de sens, ici de merveilleux, de sorte que l'auteur peut conclure que cette littérature accède, de plein droit, au statut de récit, au même titre que les œuvres écrites.

L'ouvrage de Francoeur est extrêmement dense et je n'ai pu ici qu'esquisser bien timidement un faible reflet des innovations théoriques qui s'y font jour. Ce livre constitue, à ce que je sache, l'avancée la plus audacieuse, en français, dans l'utilisation des théories de la pragmatique pour rendre compte de faits littéraires.

Reste cependant une question. Comment ces études de pragmatique, toutes orientées sur la communication s'intègrent-elles dans la sémiotique qui, elle, est principalement vouée aux problèmes de signification? La réponse n'est pas évidente et il est fort probable que l'auteur se soit lui-même posé la question. En atteste la conclusion (qui constitue, à mon goût personnel, le meilleur morceau de cet ouvrage) où la problématique est complètement déplacée: les actes culturels y sont questionnés du point de vue de la problématique de Peirce: les réseaux d'échange deviennent des rapports dialogiques entre un objet et son interprétant (ce dernier terme étant pris dans un sens trop personnalisé pour correspondre à son acceptation rigoureuse chez Peirce). Alors la culture est donnée comme un processus continu et ininterrompu d'échanges, de transformations de formes signifiantes, ce qui permet de mettre en scène une représentation de la semiosis peircienne. 
Georges Riser, dans Conjonction et disjonction dans la poésie de SaintDenys Garneau ${ }^{2}$ emprunte, à la différence de Francoeur, une démarche inductive. Les éléments de base se résument à de simples potentialités logiques: les notions de cohésion et de rupture, au regard d'une analyse formelle, deviennent des procédés sémiotiques qui ont pour effet de constituer différemment suivant leurs emplois des textes diversifiés.

Le choix de cette démarche inductive a, pour ainsi dire, forcé l'auteur à construire de façon systématique, ces notions de conjonction et de disjonction sur tous les niveaux de l'analyse linguistique, et ce sur les deux plans de l'expression et du contenu. Les deux premiers chapitres de cet ouvrage sont réservés à ce travail de construction.

Enfin le troisième chapitre est consacré aux descriptions critiques de poèmes de Saint-Denys Garneau. Et, comme toujours, c'est aux résultats que s'évalue l'appareil théorique.

Les résultats sont étonnants: ainsi dans la plupart des poèmes, la conjonction semble prédominer, particulièrement dans la structuration syntaxique où des propositions grammaticales atteignent un degré inouï de complexité, mais toujours dans le cadre d'une cohésion. Par contre, la disjonction, comme processus, apparait précisément dans le décalage entre les différents niveaux de structuration syntaxique et sémantique.

L'auteur conclut sur un rattachement idéologique de ces deux notions, la conjonction étant liée à une philosophie unitaire, intégrative du monde (christianisme, rationalité scientifique, idéologies englobantes tel le marxisme) alors que la disjonction caractériserait plutôt la modernité. C'est ainsi que ces deux notions trouvent une signification historique: chez SaintDenys Garneau, la conjonction est moins simple, moins dominante que chez les Nelligan, les Loranger et les Grandbois, alors que la disjonction reste très timide en comparaison du rôle qu'elle jouera dix ans plus tard dans la poésie d'un Paul-Marie Lapointe et d'un Gauvreau.

Dans ces conditions, la pertinence du corpus garnien en rapport avec cette problématique paraît d'autant plus juste que précisément Saint-Denys Garneau a marqué historiquement ce point de rupture au-delà duquel on ne trouvera plus que des lignes de fuite.

1. Louis Francoeur, Les Signes s'envolent. Pour une sémiotique des actes de langage culturels, Québec, 1985, P. U.L., 236 p. ( Vie des Lettres québécoises", -24).

2. Georges Riser, Conjonction et disjonction dans la poésie de Saint-Denys Garneau. Étude du fonctionnement des phénomènes de cohésion et de rupture dans des textes poétiques, Ottawa, 1984, Éd., l'U. d'Ottawa, 241 p. 